\title{
Effect of Limb Lengthening on Internodal Length and Conduction Velocity of Peripheral Nerve
}

\author{
A. Hamish Simpson, ${ }^{1}$ Thomas H. Gillingwater, ${ }^{2,3}$ Heather Anderson, ${ }^{4}$ David Cottrell, ${ }^{4 \dagger}$ Diane L. Sherman, ${ }^{4}$ \\ Richard R. Ribchester, ${ }^{3}$ and Peter J. Brophy ${ }^{4}$ \\ ${ }^{1}$ Department of Orthopaedic Surgery, ${ }^{2}$ Centre for Integrative Physiology, ${ }^{3}$ Euan MacDonald Centre for MND Research, and ${ }^{4}$ Centre for Neuroregeneration, \\ University of Edinburgh, Edinburgh EH16 4SB, United Kingdom
}

The influences of axon diameter, myelin thickness, and internodal length on the velocity of conduction of peripheral nerve action potentials are unclear. Previous studies have demonstrated a strong dependence of conduction velocity on internodal length. However, a theoretical analysis has suggested that this relationship may be lost above a nodal separation of $\sim 0.6$ mm. Here we measured nerve conduction velocities in a rabbit model of limb lengthening that produced compensatory increases in peripheral nerve growth. Divided tibial bones in one hindlimb were gradually lengthened at $0.7 \mathrm{~mm}$ per day using an external frame attached to the bone. This was associated with a significant increase $(33 \%)$ of internodal length $(0.95-1.3 \mathrm{~mm})$ in axons of the tibial nerve that varied in proportion to the mechanical strain in the nerve of the lengthened limb. Axonal diameter, myelin thickness, and $g$-ratios were not significantly altered by limb lengthening. Despite the substantial increase in internodal length, no significant change was detected in conduction velocity $(\sim 43$ $\mathrm{m} / \mathrm{s}$ ) measured either in vivo or in isolated tibial nerves. The results demonstrate that the internode remains plastic in the adult but that increases in internodal length of myelinated adult nerve axons do not result in either deficiency or proportionate increases in their conduction velocity and support the view that the internodal lengths of nerves reach a plateau beyond which their conduction velocities are no longer sensitive to increases in internodal length.

\section{Introduction}

Nerve conduction velocity depends critically on axon diameter and myelination (Arbuthnott et al., 1980). However, the significance of internodal length is unclear and has been difficult to establish experimentally, since axon diameter and myelin thickness usually covary with the internodal length (Rushton, 1951; Brill et al., 1977). Caruso et al. (1992) found slower conduction velocities in the digital nerves of fingers with internodal lengths of the order of $500 \mu \mathrm{m}$. More recently, measurements of nerve conduction velocity in periaxin-null mutant mice, in which internodal length, but not axon diameter or myelin thickness, were less than normal, showed strong dependence on internodal length (Court et al., 2004). However, as originally proposed by Huxley and Stämpfli (1949), Brill et al. (1977) predicted in their theoretical model that at long internodal lengths (such as found in large mammal and human peripheral nerve), nerve conduction velocity would become less dependent on the internodal length. We tested this prediction in the present study, using a

Received Aug. 23, 2012; revised 0ct. 26, 2012; accepted Nov. 30, 2012.

Author contributions: A.H.S., D.C., and R.R.R. designed research; A.H.S., T.H.G., H.A., D.C., D.L.S., R.R.R., and P.J.B. performed research; A.H.S., T.H.G., H.A., D.L.S., R.R.R., and P.J.B. analyzed data; A.H.S., T.H.G., D.L.S., R.R.R., and P.J.B. wrote the paper.

Work in the laboratory of P.J.B. was supported by the Wellcome Trust.

${ }^{\dagger}$ We are sad to report that David Cottrell died during the preparation of this manuscript.

This article is freely available online through the J Neurosci Open Choice option.

Correspondence should be addressed to Prof. A. Hamish Simpson at the above address. E-mail: hamish.simpson@ed.ac.uk.

DOI:10.1523/JNEUROSCI.4176-12.2013

Copyright $\odot 2013$ the authors $\quad 0270-6474 / 13 / 334536-04 \$ 15.00 / 0$ rabbit model of limb lengthening, based on procedures used in orthopedic clinical practice. We measured the responses of peripheral nerve both morphologically and physiologically. We found that peripheral nerves in lengthened limbs showed significant increases in internodal length but not conduction velocity, and with no significant pathology or changes in axon diameter, myelin thickness, or $g$-ratio. Thus, nerve conduction velocity was independent of internodal length at larger $(>0.9 \mathrm{~mm})$ nodal separations.

\section{Materials and Methods}

\section{Limb-lengthening model}

Experiments were performed on twelve 26-week-old male New Zealand White rabbits. The animals were individually caged and all surgical procedures complied with UK Home Office regulations. By 26 weeks, longitudinal growth in these rabbits is negligible (Masoud et al., 1986). Surgery was performed under deep general anesthesia (Hypnorm, 0.2 $\mathrm{ml} / \mathrm{kg}$, and Diazepam, $0.1 \mathrm{mis} / \mathrm{kg}$, supplemented with local anesthetic, $1 \%$ Lignocaine, with additional alternate doses of Hypnorm or Diazepam every 20-40 min, titrated according to veterinary guidance). The left leg of the rabbit was shaved and prepared using Hibitane. A $7 \mathrm{~cm}$ incision was made along the subcutaneous border of the tibia. The subcutaneous border of the tibia was exposed so that four screws could be inserted into the bone after predrilling with a $2 \mathrm{~mm}$ drill and a mini external fixator (Orthofix M100) applied. (Fig. 1A). A mid diaphysial osteotomy was then created just inferior to the site on the tibia where the fibula becomes conjoined. (Fig. 1A). The tibial nerve was located. Minimal dissection of the nerve was performed so that as far as possible it remained in its natural bed. The nerve was tagged in a subset of animals with fine metal rings stitched to the epineurium using a 9-0 nylon suture and loupe magnification ( $5 \times$; Fig. 1A). Following recovery from anes- 


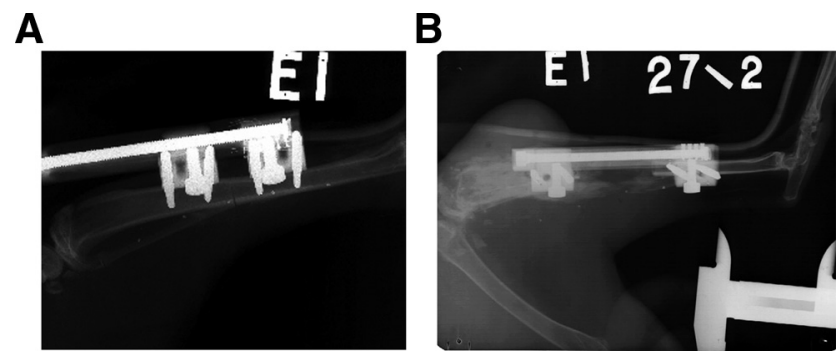

Figure 1. Radiographs of rabbit tibia with bone divided and external fixator in place. The tibial nerve is marked with metal rings approximated to the nerve with fine epineurial sutures. $\boldsymbol{A}$, Immediately postoperative. $\boldsymbol{B}$, After $30 \mathrm{~mm}$ of lengthening with early bone formation.

thesia, limbs were then lengthened by turning the screw on the external frame by half a turn $(0.33 \mathrm{~mm})$ twice per day. Nine animals underwent lengthening between 30 and $90 \mathrm{~mm}$ and three underwent a sham procedure: that is, the osteotomy was created, the external fixator applied but they did not undergo any lengthening. Once the lengthening had been completed, a consolidation period of $\sim 1$ month was allowed for the soft tissues to adapt to their new length.

\section{Electrophysiology}

In vivo measurements. Under general anesthesia (Hypnorm and Diazepam), two pairs of cutaneous stimulating electrodes with a fixed separation of $90 \mathrm{~mm}$ were placed in a thermoplastic splint constructed to follow the shape of the posteromedial half of the leg, which stimulated the tibial nerve on the medial aspect of the calf just distal to the knee joint and posterior to the medial malleolus. A $1 \mathrm{~ms}$ square wave pulse was applied; the amplitude was increased until the toes were seen to twitch. Recordings were made from the intrinsic muscles in the sole of the foot using surface electrodes, after preparing the skin with shaving and cleaning.

The difference in the latencies from the stimulus artifact to the peak for stimulation points at the knee and medial malleolus were recorded and used to calculate the conduction velocity.

In vitro measurements. Animals were killed by overdose of sodium pentobarbitone ( $150 \mathrm{mg} / \mathrm{kg}$ body weight) (Euthatal, Merial, UK)The tibial nerves of both hind limbs were dissected and placed into oxygenated mammalian physiological solution (in mM: $120 \mathrm{NaCl}, 5 \mathrm{KCl}, 2 \mathrm{CaCl}_{2}, 1$ $\mathrm{MgCl}_{2}, 0.4 \mathrm{NaH}_{2} \mathrm{PO}_{4}, 23.8 \mathrm{NaHCO}_{3}, 5.6$ D-glucose) equilibrated with $5 \% \mathrm{CO}_{2} / 95 \% \mathrm{O}_{2}$ (Ribchester et al., 2004). They were then transferred to a recording chamber maintained at room temperature $\left(23^{\circ} \mathrm{C}\right.$ approximately) containing an array of silver electrodes spaced at $0.5 \mathrm{~cm}$ intervals. The proximal end of the nerve was stimulated with rectangular pulses $(0.1 \mathrm{~ms}, 0.1-5 \mathrm{~V})$ and the conduction distance was varied from 2 to $7 \mathrm{~cm}$ by altering the position of the stimulating electrode. Compound action potentials were recorded at room temperature using a Powerlab interface (AD Instruments), digitized and analyzed using Chart and Scope software on Apple Macintosh or PC computers.

The modal conduction velocity of Group A- $\alpha$ axons was estimated from the slope of the relationship between the distance from the cathode and the proximal recording electrode, and the latency from stimulus artifact to the first peak of the compound action potential.

\section{Internodal length measurements}

Following death by sodium pentobarbitone overdose, tibial nerves were dissected and fixed overnight in $4 \%$ paraformaldehyde, $0.1 \mathrm{~m}$ sodium phosphate buffer, $\mathrm{pH} 7.4$, washed in several changes of phosphate buffer, and teased using acupuncture needles on 3-aminopropyltriethoxysilane (TESPA)-coated slides. The samples were mounted with Vectashield containing DAPI $(1.5 \mu \mathrm{g} / \mathrm{ml})$ and imaged using a $10 \times$ objective lens on an Olympus fluorescence microscope (BX60). Images were captured on an Orca-ER camera (Hamamatsu Photonics) using Openlab software (PerkinElmer). Montages were assembled using Photoshop CS4 software and internodal lengths (mean $47.5 \pm 11.3$ per nerve) were measured using Openlab software.

\section{Electron microscopy}

Tibial nerves were processed for electron microscopy as previously described (Comley et al., 2011). In brief, following death by sodium pentobarbitone overdose, nerves were rapidly dissected before fixation in $0.1 \mathrm{M}$ phosphate buffer containing 4\% paraformaldehyde and $2.5 \%$ glutaraldehyde for $4 \mathrm{~h}$ and postfixation in 1\% osmium tetroxide for $45 \mathrm{~min}$. Following dehydration through an ascending series of ethanol solutions and propylene oxide, preparations were embedded in Durcupan resin. Ultrathin sections $(\sim 70 \mathrm{~nm})$ were collected on Formvar-coated grids (Agar Scientific), stained with uranyl acetate and lead citrate and then viewed in a Philips CM12 transmission electron microscope equipped with a Gatan digital camera. Morphometric measurements (including axon diameter and myelin sheath thickness) were made from digital micrographs using ImageJ software with data collected and analyzed using Microsoft Excel and GraphPad Prism software. For each individual fiber, axon diameters and $G$ ratios were calculated as previously described (Sherman et al., 2012).

\section{Statistical analysis}

Data were checked for normality and then Student's $t$ tests were used. The experimental study design and the number of recordings indicated $>99 \%$ power to detect a $40 \%$ difference in conduction velocity (that is, a conduction velocity that varies in direct proportion to internodal length) or $80 \%$ power to detect a difference in conduction velocity of only $20 \%$ (GraphPad StatMate).

\section{Results}

All experimental animals showed significant limb lengthening with evidence of new bone formation. The amount of lengthening was clearly visible on $\mathrm{x}$-ray (Fig. 1B).

Isolated, teased axons had a normal appearance, with no evidence of Wallerian degeneration or demyelination in any of the nerves dissected from the lengthened limbs or the controls. The internodal length was greater by $\sim 33 \%$ (control $0.93 \pm 0.1 \mathrm{~mm}$; lengthened $1.27 \pm 0.03 \mathrm{~mm}$, mean $\pm \mathrm{SEM}, n=3, p<0.05, t$ test (Fig. $2 B$ ). The axon and myelin diameters, and $g$-ratios were measured from the electron microscope images. The axonal diameters (control: $3.42 \pm 0.84 \mu \mathrm{m}$; lengthened: $3.20 \pm 0.6 \mu \mathrm{m}$ ) and the $g$-ratio (control: $0.59 \pm 0.02$; lengthened: $0.63 \pm 0.04$ ) were not significantly changed by the lengthening.(Fig. 2C,D). The percentage increase in internodal length closely matched the nerve strain measured with the metallic radiographic markers on $\mathrm{x}$-ray $(26.1 \%$ strain on $\mathrm{x}$-ray vs $23.6 \%$ increase in internodal length, $67.3 \%$ strain on $\mathrm{x}$-ray vs $69.4 \%$ increase in internodal length).

Nerve conduction velocity was measured in vivo at the end of lengthening. The in vivo measurements showed no change in conduction velocity $(40.3 \pm 0.48 \mathrm{~m} / \mathrm{s}$ post-lengthening, $41.5 \pm$ $1.5 \mathrm{~m} / \mathrm{s}$ controls $n=4, p=0.46$ ). After the accommodation period of 1 month following the completion of lengthening, which allowed the tissues to adapt to the new length of the limb, measurements of conduction velocity in vitro in a further 5 animals showed a mean of $37.4 \pm 3.8 \mathrm{~m} / \mathrm{s}$ in the control limbs that was not significantly different in the lengthened limbs $(32.7 \pm 1.6$ $\mathrm{m} / \mathrm{s}, n=5, p=0.22$ ) (Fig. $3 A, B$ ). Overall, the combined ex vivo and in vivo measurements showed conduction velocities of $39.2 \pm 2.2 \mathrm{~m} / \mathrm{s}$ in the controls and $36.0 \pm 1.6 \mathrm{~m} / \mathrm{s}$ in the lengthened limbs. ( $n=9, p=0.15$ ) (Fig. 3C).

The conduction velocity did not change significantly in shamoperated control animals $(43 \pm 1.2 \mathrm{~m} / \mathrm{s}$ in the controls limbs, $44 \pm 1.7 \mathrm{~m} / \mathrm{s}$ in the sham-operated limbs, mean $\pm \mathrm{SEM}, n=3$, $p=0.66)$.

\section{Discussion}

In this model of limb lengthening, we have demonstrated that peripheral nerves increased in length and that this was accompa- 
nied by an increase in length of the internodes, yet the axons did not narrow significantly. Our results are consistent with more axoplasm and myelin being generated purely in response to a gradual distraction stimulus. Previously a similar phenomenon of tissue histiogenesis has been reported in muscle in response to a lengthening stimulus (Simpson et al., 1995).

The model we used is based on the procedures performed for limb lengthening in clinical practice. Until the 1980s, surgical procedures primarily relied on stretching of the soft tissues rather than harnessing the biological potential of the tissues to respond to an appropriate incremental distraction. For instance, Ilizarov and Deviatov (1969) reported that if a bone was divided and the two bone fragments stabilized for 1 week and then slowly pulled apart at a rate of $1 \mathrm{~mm}$ per day or less, the body responded by creating a column of new bone. This technique is termed distraction osteogenesis and is extremely successful in creating new bone purely in response to a gradual lengthening stimulus. This study examines the response of peripheral nerve tissue to the same gradual lengthening stimulus.

As a human grows the number of nodes of Ranvier stays the same (Vizoso and Young, 1948; Jacobs, 1988) but the length of the internodes increases in a linear fashion (Williams and WendellSmith, 1971). This has also been confirmed in mouse development (Court et al., 2004). In this study we found that there was a significant increase in the internodal length and that the axon diameter and the myelin thickness and hence the $g$-ratio remained unchanged.

Thus, the internodal lengths for the rabbit, the mouse and the human behave similarly and increase in length in line with the growth of the limb. These results indicate how even in the adult animal, the internode/myelin sheath retains its ability to change and that it will continue to respond to a lengthening stimulus in a similar way to the changes that occur during growth. Similar findings of an increase in internodal length with lengthening were obtained by Abe et al. $(2002,2004)$ in a rat model. However in contrast to their results, we did not observe pathological changes. Abe et al. $(2002,2004)$ used rates of lengthening of the sciatic nerve of $1.6 \%$ per day (Abe et al., 2004) and 5-10\% per day (Abe et al., 2002). In contrast our rate of lengthening of $0.5 \%$ per day was closer to the rates of lengthening that occurs during normal growth $(0.2 \%$ per day in first postnatal year in humans).

In previous studies we have found that conduction velocities are strongly influenced by internodal length in fibers over a range of short internodal lengths between 0.2 and $0.6 \mathrm{~mm}$ in genetically modified mice (Court et al., 2004). In the current work, internodal length was increased by $\sim 35 \%$ with limb lengthening $(p<$ 0.5 ; $t$ test) but there was no discernible or statistically significant change in conduction velocity $(p>0.05)$. That is, we observed that conduction velocity was not significantly altered when inter- nodal length varied between 1.0 and $1.4 \mathrm{~mm}$. Thus, over the range of increase in internodal length induced by limb lengthening and at this level of statistical power, conduction velocity appeared independent of mean internodal length. Regardless of the validity of the null hypothesis, however, it was evident that nerve conduction was unimpaired by the increase in internodal length, as demonstrated by the persistence of healthy, high-velocity compound action potentials after limb lengthening.

Our findings concur with the studies of Whitteridge and Sanders (1946) who reported that after the crush of the peroneal nerve in the rabbit, the internodal length which initially was $\sim 1.1$ $\mathrm{mm}$ decreased to approximately half that of the contralateral nerve, yet at $\sim 450 \mathrm{~d}$, after the crush injury the conduction velocity had returned to the same value as the opposite limb.

The results are also consistent with the assertion of Huxley and Stämpfli (1949) that the conduction velocity would increase as the internodal length increased until a "flat maximum" was reached and the theoretical analysis reported by Brill et al. (1977) suggesting that at internodal lengths $>600 \mu \mathrm{m}$, the conduction velocity becomes independent of the internodal length, as a consequence of the increased capacitance of the internode as its length increases. The proposal of Huxley and Stämpfli (1949) of the "flat maximum" has recently received experimental support from studies on a mouse mutant (Wu et al., 2012). Our results are 
also consistent with data reported on the conduction velocity at different ages in children, which demonstrate a linear increase during the first year and a plateau from 5 year to skeletal maturity. According to the findings of Jacobs (1988) and Williams and Wendell-Smith (1971), during this period the internodal length would have increased in line with limb growth. In the immature rabbit the internodal length has been reported to be $\sim 0.2 \mathrm{~mm}$ (Williams and Wendell-Smith, 1971). In humans, at birth, 1 year, 5 years, and at maturity, the lower limb length is approximately $15,25,42$, and $81 \mathrm{~cm}$, respectively (Kelly and Diméglio, 2008). Thus in the first year there would have been a $66 \%$ increase in internodal length and an estimated $60 \%$ increase in conduction velocity has been observed (García et al., 2000), which is in keeping with the conduction velocity being proportional to the internodal length at this stage. However, between, 5 years and skeletal maturity a $93 \%$ increase in internodal length would be expected yet the conduction velocity is reported to have reached adult levels by this age (Cai and Zhang, 1977) or for there to be a small increase from age 5 to skeletal maturity (approx 15\%) (García et al., 2000). Thus the clinical data are consistent with our results and the theoretical analysis reported by Brill et al. (1977).

In summary, we report the response of peripheral nerves in a clinically relevant model of limb lengthening. Our results are consistent with nerve tissue histiogenesis occurring purely in response to a lengthening stimulus. A significant increase in the internodal length was observed without a change in the axon diameter, myelin thickness or g-ratio, demonstrating that the myelin sheath/internode retained its adaptive plasticity even in the adult. Despite the significant increase in internodal length, the conduction velocity remained unchanged, which provides experimental confirmation of the theoretical assertion that at larger values of the internodal length, the conduction velocity is no longer proportional to the internodal length but becomes independent of it.

\section{References}

Abe I, Tsujino A, Hara Y, Ichimura H, Ochiai N (2002) Paranodal demyelination by gradual nerve stretch can be repaired by elongation of internodes. Acta Neuropathol 104:505-512. Medline

Abe I, Ochiai N, Ichimura H, Tsujino A, Sun J, Hara Y (2004) Internodes can nearly double in length with gradual elongation of the adult rat sciatic nerve. J Orthop Res 22:571-577. CrossRef Medline

Arbuthnott ER, Boyd IA, Kalu KU (1980) Ultrastructural dimensions of myelinated peripheral nerves in the cat and their relation to conduction velocity. J Physiol 308:125-157. Medline

Brill MH, Waxman SG, Moore JW, Joiner RW (1977) Conduction velocity and spike configuration in myelinated fibres: computed dependence on internode distance. J Neurol Psychiatry 40:769-774. CrossRef

Cai F, Zhang J (1997) Study of nerve conduction and late responses in normal Chinese infants, children, and adults. J Child Neurol 12:13-18. CrossRef Medline

Caruso G, Massini R, Crisci C, Nilsson J, Catalano A, Santoro L, Battaglia F, Crispi F, Nolano M (1992) The relationship between electrophysiological findings, upper limb growth and histological features of median and ulnar nerves in man. Brain 115:1925-1945. CrossRef Medline

Comley LH, Fuller HR, Wishart TM, Mutsaers CA, Thomson D, Wright AK, Ribchester RR, Morris GE, Parson SH, Horsburgh K, Gillingwater TH (2011) ApoE isoform-specific regulation of regeneration in the peripheral nervous system. Hum Mol Genet 20:2406-2421. CrossRef Medline

Court FA, Sherman DL, Pratt T, Garry EM, Ribchester RR, Cottrell DF, Fleetwood-Walker SM, Brophy PJ (2004) Restricted growth of Schwann cells lacking Cajal bands slows conduction in myelinated nerves. Nature 431:191-195. CrossRef Medline

García A, Calleja J, Antolín FM, Berciano J (2000) Peripheral motor and sensory nerve conduction studies in normal infants and children. Clin Neurophysiol 111:513-520. CrossRef Medline

Huxley AF, Stämpfli R (1949) Evidence for saltatory conduction in peripheral myelinated nerve fibres. J Physiol 108:315-339. Medline

Ilizarov GA, Deviatov AA (1969) [Surgical lengthening of the shin with simultaneous correction of deformities.] Ortop Travmatol Protez 30: 32-37. Medline

Jacobs JM (1988) On internodal length. J Anat 157:153-162. Medline

Kelly PM, Diméglio A (2008) Lower-limb growth: how predictable are predictions? J Child Orthop 2:407-415. CrossRef Medline

Masoud I, Shapiro F, Kent R, Moses A (1986) A longitudinal study of the growth of the New Zealand White Rabbit: Cumulative and biweekly incremental growth rates for body length, body weight, femoral length and tibial length. J Orthop Res 4:221-231. CrossRef Medline

Ribchester RR, Thomson D, Wood NI, Hinks T, Gillingwater TH, Wishart TM, Court FA, Morton AJ (2004) Progressive abnormalities in skeletal muscle and neuromuscular junctions of transgenic mice expressing the Huntington's disease mutation. Eur J Neurosci 20:3092-3114.

Rushton WAH (1951) A theory of the effects of fibre size in medullated nerve. J Physiol 115:101-122. Medline

Sherman DL, Krols M, Wu LM, Grove M, Nave KA, Gangloff YG, Brophy PJ (2012) Arrest of myelination and reduced axon growth when Schwann cells lack mTOR. J Neurosci 32:1817-1825.

Simpson AH, Williams PE, Kyberd P, Goldspink G, Kenwright J (1995) The response of muscle to leg lengthening. J Bone Joint Surg Br 77:630-636.

Vizoso AD, Young JZ (1948) Internode length and fibre diameter in developing and regenerating nerves. J Anat 82:110-135. Medline

Whitteridge D, Sanders FK (1946) Conduction velocity and myelin thickness in regenerating nerve fibres. J Physiol 105:152-174. Medline

Williams PL, Wendell-Smith CP (1971) Some additional parametric variations between peripheral nerve fibre populations. J Anat 109:505-526. Medline

Wu LMN, Williams A, Delaney A, Sherman DL, Brophy PJ (2012) Increasing internodal distance in myelinated nerves accelerates nerve conduction to a flat maximum. Curr Biol 22:1957-1961. CrossRef Medline 